\title{
Simulador Computacional para Demonstração das Propriedades um Gás
} Ideal em 2 ${ }^{+*}$

Luciano Frontino de Medeiros ${ }^{1}$

Álvaro Crovador ${ }^{1}$

Programa de Pós-Graduação em Educação e Novas Tecnologias

Centro Universitário Internacional UNINTER

Curitiba - PR

Hamilton Pereira da Silva ${ }^{1}$

Departamento de Ciência da Computação

Universidade Federal Tecnológica do Paraná

Medianeira - PR

\section{Resumo}

Este artigo apresenta um simulador computacional para demonstrar as propriedades de um gás ideal bidimensional, a partir da dedução da equação de estado para o caso de duas dimensões, permitindo visualizar como a partir do fenômeno microscópico das colisões se obtém as grandezas macroscópicas do gás. São mostradas as equivalências entre as abordagens $2 D$ e 3D, bem como a distribuição de Maxwell-Boltzmann modificada para o caso 2D. É descrito o funcionamento do simulador construído em Javascript/HTML, a mensuração das grandezas para o caso $2 \mathrm{D}$ bem como uma análise adicional do comportamento das colisões das partículas com as paredes do recipiente em $2 D$, mostrando que a quantidade de colisões com as paredes não é constante no decorrer da simulação.

Palavras-chave: Gás Bidimensional; Termodinâmica; Simulador Computacional; Equação de Estado de um Gás Ideal.

\footnotetext{
${ }^{+}$Computational Simulator for Demonstrating the Properties of a 2D Ideal Gas

* Recebido: agosto de 2017. Aceito: junho de 2018.

${ }^{1}$ E-mails: luciano.me@uninter.com; alvaro.c@uninter.com; hpsilva@utfpr.edu.br
} 


\begin{abstract}
This paper presents a computational simulator to demonstrate properties of a two-dimensional ideal gas from the equation of state for $2 \mathrm{D}$ case, allowing to view the obtaining of macroscopic quantities from the microscopic perspective of collisions according to Newton's law. An equivalence between $2 D$ and $3 D$ approaches are available, as well as the Maxwell-Boltzmann velocities distribution modified for 2D. The paper describes some operational aspects in Javascript/HTML, the measure units for $2 D$ case and a further analysis of collisions behavior with the recipient $2 D$ borders, showing that the number of collisions with the borders isn't a constant along the simulation.
\end{abstract}

Keywords: Two-dimensional Gas; Thermodynamics; Computational Simulation; Equation of State of an Ideal Gas.

\title{
I. Introdução
}

O estudo da Termodinâmica teve um papel fundamental no desenvolvimento da visão corpuscular da matéria ao final do século XIX, sendo considerada por alguns o embrião da ideia do quantum no alvorecer do século XX. O edifício da teoria cinética dos gases foi construído a partir dos estudos e experimentos de uma série de personalidades, dentre as quais Robert Boyle (1627-1691), Jacques Charles (1746-1823), Louis J. Gay-Lussac (1778-1850), Benoit Clapeyron (1799-1864) para o relacionamento das variáveis macroscópicas dos fenômenos termodinâmicos e, por fim, James C. Maxwell (1831-1879) e Ludwig V. Boltzmann (1844-1906), na demonstração da conexão entre a visão microscópica das velocidades das moléculas com a variável macroscópica da temperatura. A noção de equipartição da energia de Boltzmann foi utilizada mais tarde para a expressão da radiação do corpo negro de Planck, o que culminou na ideia do tratamento da energia como uma grandeza discreta ao invés de contínua (EISBERG; RESNICK, 1979, p. 33).

A construção de simuladores que permitam mostrar como os fenômenos físicos se comportam em uma "caixa de areia" é de grande valia para o ensino de Física e Matemática. Conforme Medeiros e Medeiros (2002, p. 79), as simulações podem ser vistas como representações ou modelagens de objetos específicos reais ou imaginados, sejam de sistemas ou fenômenos. A partir da visualização da evolução de um fenômeno em simuladores, os alunos podem compreender como é o comportamento das diferentes grandezas ligadas ao fenômeno físico. No sentido da compreensão de como os fenômenos microscópicos geram os macroscópicos, o uso de simuladores que possam demonstrar como, a partir das colisões entre moléculas, emerge o conceito da energia e da temperatura, é uma justificativa importante para o desenvolvimento de simuladores e objetos de aprendizagem para a área da Termodinâmica. 
Para simplificar a construção do simulador, este trabalho tem o foco na utilização do conceito de gás bidimensional, para tratar o problema em duas dimensões. Para se chegar a uma equação que descreva o estado de um gás ideal em duas dimensões, é oportuna a descrição de um modelo que produza a equação de estado de um gás em três dimensões. A equação de estado que rege um gás em 3D relaciona a pressão, o volume que o gás ocupa e a temperatura, o qual se constata a partir da equação de Clapeyron e das relações estabelecidas por Boyle, Charles e Gay-Lussac entre as três variáveis macroscópicas. Por meio de uma analogia da descrição em duas dimensões, pode-se chegar também a um correlato da equação de Clapeyron em $2 \mathrm{D}$, considerando a área ocupada pelo gás, em vez do volume.

A noção do gás ideal em duas dimensões possui uma extensa utilização em pesquisas envolvendo o confinamento de partículas supostas como um gás em duas dimensões. Por exemplo, o comportamento de moléculas de benzeno em uma superfície de cobre a baixa temperatura pode ser detectado por meio de um microscópio de tunelamento (STRANICK; KAMNA; WEISS, 1994). O gás de elétrons em duas dimensões é um modelo utilizado em física de estado sólido, onde o confinamento leva a estados de energia quantizados para o movimento na terceira dimensão. A explicação do movimento dos elétrons em um cristal de grafeno pode ser ajustada à noção do gás de elétrons em 2D (NOVOSELOV et al., 2012). A equipartição de energia de um gás em duas dimensões também é objeto de simulação (SCHERER, 2005, p. 195-206). Também se encontram pesquisas considerando fluidos entre duas placas paralelas, cujo formalismo é descrito como uma forma intermediária entre duas e três dimensões (BREY; MAYNAR; GARC, 2016).

Simulações envolvendo gás ideal em 2D como objeto de pesquisa pode ser evidenciado em alguns trabalhos. Aiello-Nicosia e Sperandeo-Mineo (1985) apresentaram um modelo de simulação de um gás bidimensional, assumindo colisões elásticas e utilizando um método de dinâmica molecular. O interesse educacional da simulação era o de demonstrar a obtenção da distribuição de Maxwell-Boltzmann utilizando apenas o framework da mecânica newtoniana. Novak e Bortz (1970) estudaram por meio da simulação o comportamento da função de distribuição de velocidade com relação ao tempo. Bonomo e Riggi (1984) ressaltaram a obtenção da distribuição de Maxwell-Boltzmann a partir de uma condição inicial onde todas as partículas tinham a mesma velocidade. Uma conclusão comum a estes trabalhos é a demonstração da natureza estatística da segunda lei da termodinâmica.

Portanto, este artigo tem como motivação a construção de um objeto de aprendizagem que permita aos alunos compreenderem a transição do modelo newtoniano de colisões microscópicas para o fenômeno termodinâmico macroscópico. Inicialmente, detalha-se a obtenção das equações para o gás ideal em 2D, fazendo-se a analogia da obtenção das grandezas em 3D para 2D, fornecendo então a base teórica para a concepção do simulador. Na sequência, detalha-se a modelagem e construção do simulador, mostrando-se alguns resultados e uma discussão a respeito dos dados obtidos, bem como uma análise das colisões com as bor- 
das da caixa 2D. As considerações finais indicam direções para estudos mais aprofundados e novas propostas de simulações.

\section{Equação de Estado de um Gás Ideal em 3D}

Para se analisar a forma como as grandezas macroscópicas surgem a partir da abordagem microscópica do problema, com base na mecânica clássica, a abstração do gás ideal leva em conta algumas suposições (CARUSO; OGURI, 2006; RESNICK; HALLIDAY, 1982, p. 210-211):

1. Um gás é constituído de partículas denominadas de moléculas.

2. As moléculas estão em movimento desordenado obedecendo às leis da mecânica newtoniana.

3. O numero de moléculas presentes é muito grande.

4. O volume que é ocupado pelas moléculas é insignificante comparado ao volume onde o gás está contido.

5. As forças intermoleculares são desprezíveis, a não ser no caso de colisão.

6. As colisões são elásticas e com duração insignificante.

A partir destas premissas, é possível deduzir a equação de estado de um gás ideal, a partir do movimento caótico molecular chegando-se a uma interpretação cinética das grandezas termodinâmicas tais como pressão e temperatura. $\mathrm{O}$ desenvolvimento das equações de Clapeyron e da energia média das moléculas de um gás ideal a seguir descrita segue principalmente Caruso e Oguri (2006, p. 69-72) e Tipler e Mosca (2014, p. 583-584). A Fig. 1 demonstra um elemento de área A no qual estão presentes as moléculas de um gás ideal.

Considerando $\mathrm{N}_{\mathrm{i}}$ a totalidade das moléculas que tem velocidades $\mathrm{v}_{\mathrm{i}}, \mathrm{N}_{\mathrm{i}} \mathrm{V}$ representa a densidade de moléculas em todo o cubo cujo volume é $\mathrm{V}$. Em um intervalo de tempo $\Delta \mathrm{t}$, as moléculas com a componente $\mathrm{v}_{\mathrm{ix}}$ na direção $\mathrm{x}$ que colidem com a parede $\mathrm{A}$ transversal à direção x estão dentro do elemento de volume dado por

$$
\Delta V=A v_{i x} \Delta t
$$

Pode-se contar o número de moléculas que se chocam contra uma das paredes do cubo, de forma transversa ao eixo $x$ no intervalo de tempo $\Delta t$, sendo

$$
N_{i x}=\frac{1}{2} \frac{N_{i}}{V} v_{i x} A \Delta t
$$

A presença do fator $1 / 2$ indica que somente metade das moléculas tem velocidades no sentido da parede $A$. 


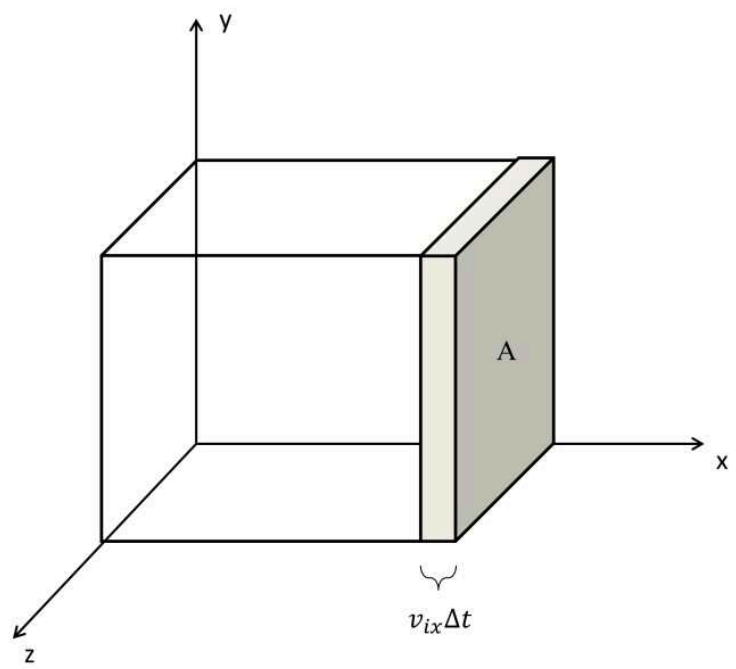

Fig. 1 - Ilustração do volume onde estão distribuídas as moléculas de um gás ideal. Adaptado de Caruso e Oguri (2006, p. 68).

As moléculas estão em colisões elásticas com a parede, sendo refletidas de tal modo que a variação da componente do momentum na direção de $x$ é dada por

$$
\Delta p_{i x}=2 m v_{i x}
$$

Portanto, a força que as moléculas exercem colidindo com a parede é

$$
F_{i x}=\frac{\Delta p_{i x}}{\Delta t}
$$

Para calcular a pressão média $P$, deve-se considerar a força total que as $N$ moléculas exercem quando as mesmas se chocam contra a parede, dividida pela área

$$
P=\sum_{i=1}^{N} N_{i x} \frac{F_{i x}}{A}=\sum_{i=1}^{N} N_{i x} \frac{\Delta p_{i x}}{A \Delta t}=\frac{m}{V} \sum_{i=1}^{N} N_{i x} v_{i x}^{2}
$$

Simplificando, obtém-se

$$
P=\frac{N}{V} m\left\langle v_{x}^{2}\right\rangle
$$

na qual $N$ é o número total de moléculas e a expressão

$$
\left\langle v_{x}^{2}\right\rangle=\frac{1}{N} \sum_{i=1}^{N} N_{i} v_{i x}^{2}
$$

refere-se à velocidade média dos quadrados das componentes de velocidade na direção $x$. Conforme o princípio do caos molecular, como as moléculas se deslocam em qualquer uma das direções com a mesma probabilidade, os valores médios quadráticos das componentes das velocidades em qualquer direção são iguais, ou seja,

$$
\left\langle v_{x}^{2}\right\rangle=\left\langle v_{y}^{2}\right\rangle=\left\langle v_{z}^{2}\right\rangle
$$

e a velocidade média dos quadrados das velocidades das moléculas do gás dada por 


$$
\left\langle v^{2}\right\rangle=\left\langle v_{x}^{2}\right\rangle+\left\langle v_{y}^{2}\right\rangle+\left\langle v_{z}^{2}\right\rangle=\frac{1}{N} \sum_{i=1}^{N} N_{i} v_{i}^{2} .
$$

A partir desta expressão, pode-se escrever

$$
\left\langle v^{2}\right\rangle=3\left\langle v_{x}^{2}\right\rangle
$$

Finalmente, a pressão do gás ideal é dada pela expressão de Joule

$$
P=\frac{1}{3} \frac{N}{V} m\left\langle v^{2}\right\rangle
$$

Uma vez que $N m$ é a massa $M$ do gás, a expressão pode ser reescrita por meio da densidade $\rho$

$$
P=\frac{1}{3} \rho\left\langle v^{2}\right\rangle
$$

As experiências de Boyle e Charles, com relação aos processos isométricos e isotérmicos, indicam que a pressão $P$, o volume $V$ e a temperatura $T$ de um gás em equilíbrio termodinâmico estão relacionadas como

$$
\frac{P V}{T}=\text { constante }
$$

A relação anterior, generalizada por Clapeyron, é expressa na forma

$$
P V=n R T
$$

sendo $n$ o número de mols do gás, calculado por $N / N_{A}$ (onde $N_{A}=6,023.10^{23}$ ) e $R \cong$ 8,315 J. $\mathrm{K}^{-1} \cdot \mathrm{mol}^{-1}$ a constante universal dos gases.

Escrevendo-se a equação de Joule (1) como

$$
P=\frac{1}{3} \frac{N}{V}\langle p v\rangle=\frac{2}{3} \frac{N}{V}\langle\epsilon\rangle=\frac{2 U}{3 V}
$$

em que $\langle\epsilon\rangle$ é a energia cinética das moléculas e $U=N\langle\epsilon\rangle$ a energia interna do gás, a equção de Clapeyron (1) permite relacionar a energia interna do gás à temperatura

$$
U=\frac{3}{2} P V
$$

Combinando-se com a equação de Clapeyron,

$$
U=\frac{3}{2} n R T=\frac{3}{2} \frac{N R T}{N_{A}}=\frac{3}{2} N k T,
$$

A quantidade $k$ obtida pela razão entre a constante dos gases e o número de Avogadro, ou seja,

$$
k=\frac{R}{N_{A}}=1,380 \cdot 10^{-23} \mathrm{~J} \cdot K^{-1} .
$$

Por fim, a expressão final da energia cinética média das moléculas é dada por, 


$$
\langle\epsilon\rangle=\frac{3}{2} k T
$$

\section{A Equação de Estado em 2D}

Para se obter as expressões que irão reger as relações entre as variáveis termodinâmicas para o caso em duas dimensões, suprime-se uma dimensão a partir da modelagem do recipiente que agora é um retângulo, sendo a parede substituída pelo lado $L$ onde as moléculas em duas dimensões se chocam, conforme a Fig. 2. No caso bidimensional, a grandeza análoga à pressão é dada pela razão força/comprimento, ou seja, uma tensão superficial.

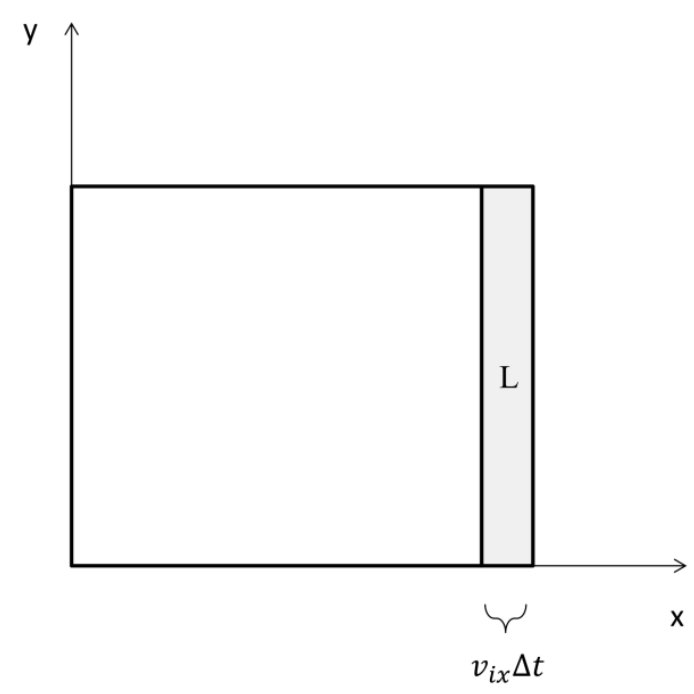

Fig. 2 - Ilustração da área onde estão distribuídas as moléculas de um gás ideal bidimensional.

Assim, o elemento de área que contém as moléculas que colidem em um intervalo de tempo $\Delta t$ é igual a

$$
\Delta A=L v_{i x} \Delta t
$$

e o número das moléculas bidimensionais agora é dado por

$$
N_{i x}=\frac{1}{2} \frac{N_{i}}{A} v_{i x} L \Delta t
$$

Como a relação do momentum mantém-se inalterada, a pressão média em 2D será agora em relação à área, ao invés do volume.

$$
P=\frac{N}{A} m\left\langle v_{x}^{2}\right\rangle
$$

Mantendo-se a hipótese do caos molecular, temos agora o princípio válido em duas dimensões

$$
\left\langle v_{x}^{2}\right\rangle=\left\langle v_{y}^{2}\right\rangle
$$




$$
\left\langle v^{2}\right\rangle=2\left\langle v_{x}^{2}\right\rangle
$$

Assim, em 2D, a expressão análoga de Boyle é dada por

$$
P=\frac{1}{2} \frac{N}{A} m\left\langle v^{2}\right\rangle
$$

Ou também, usando-se a densidade em duas dimensões $\rho=m / A$,

$$
P=\frac{1}{2} \rho\left\langle v^{2}\right\rangle
$$

Modificando-se a equação análoga de Clapeyron

$$
P A=n R T,
$$

A expressão da energia interna anula o divisor 2 da expressão original em 3D de $U$, modificando para

$$
U=P A,
$$

o que simplifica a expressão da energia cinética média das moléculas para

$$
\langle\epsilon\rangle=k T .
$$

Portanto, tem-se de forma análoga à derivação em 3D as fórmulas para descrever o comportamento de um gás ideal em duas dimensões. Da equação (2) decorre a relação entre a velocidade média das partículas e a temperatura do gás bidimensional

$$
\frac{m\left\langle v^{2}\right\rangle}{2}=k T \therefore\langle v\rangle=\sqrt{\frac{2 k T}{m}} .
$$

A seguir, a analogia é estendida para se obter a distribuição das velocidades.

\section{A Distribuição 2D de Maxwell-Boltzmann}

A dedução da equação de Maxwell-Boltzmann, que descreve a distribuição dos módulos das velocidades quadráticas médias das moléculas, é feita levando-se em consideração uma caixa contendo um gás ideal, cujas moléculas se chocam em colisões perfeitamente elásticas. Enquanto que em uma componente, a distribuição de velocidade é (CARUSO; OGURI, 2006, p. 76-77; TIPLER; MOSCA, 2014, p. 589-590)

$$
f_{x}\left(v_{x}^{2}\right)=\left(\frac{m}{2 \pi k T}\right)^{\frac{1}{2}} \exp \left(-\frac{1}{2} \frac{m v_{x}^{2}}{k T}\right)
$$

A distribuição das velocidades de Maxwell é dada por

$$
f\left(v^{2}\right)=\left(\frac{m}{2 \pi k T}\right)^{\frac{3}{2}} \exp \left(-\frac{1}{2} \frac{m v^{2}}{k T}\right)
$$


A partir da seguinte igualdade, a distribuição dos módulos pode ser calculada por

$$
f\left(v^{2}\right) d v_{x} d v_{y} d v_{z}=f\left(v^{2}\right) v^{2} d v \sin \theta d \theta d \phi .
$$

Mas $f\left(v^{2}\right)$ não depende das coordenadas polares. Portanto, integrando com relação aos ângulos $\theta$ e $\phi$, a distribuição dos módulos das velocidades será dada por

$$
p(v)=\sqrt{\frac{2}{\pi}}\left(\frac{m}{k T}\right)^{\frac{3}{2}} v^{2} \exp \left(-\frac{1}{2} \frac{m v^{2}}{k T}\right)
$$

Analogamente, a distribuição 2D de Maxwell se modifica para

$$
f\left(v^{2}\right)=\left(\frac{m}{2 \pi k T}\right) \exp \left(-\frac{1}{2} \frac{m v^{2}}{k T}\right) .
$$

Em duas dimensões pode-se escrever a igualdade

$$
f\left(v^{2}\right) d v_{x} d v_{y}=f\left(v^{2}\right) v d v d \theta
$$

Com integração em $\theta$, a distribuição dos módulos em 2D resulta como

$$
p(v)=\frac{m}{k T} v \exp \left(-\frac{1}{2} \frac{m v^{2}}{k T}\right)
$$

Na Fig. 3, pode-se comparar a influência do fator $v$ na distribuição 2D para o fator em $v^{2}$ na distribuição 3D. Na Fig. 4, é mostrado o gráfico da distribuição 2D para três diferentes temperaturas. As velocidades no eixo $x$ de ambos os gráficos são expressas em unidades arbitrárias (u.a.).

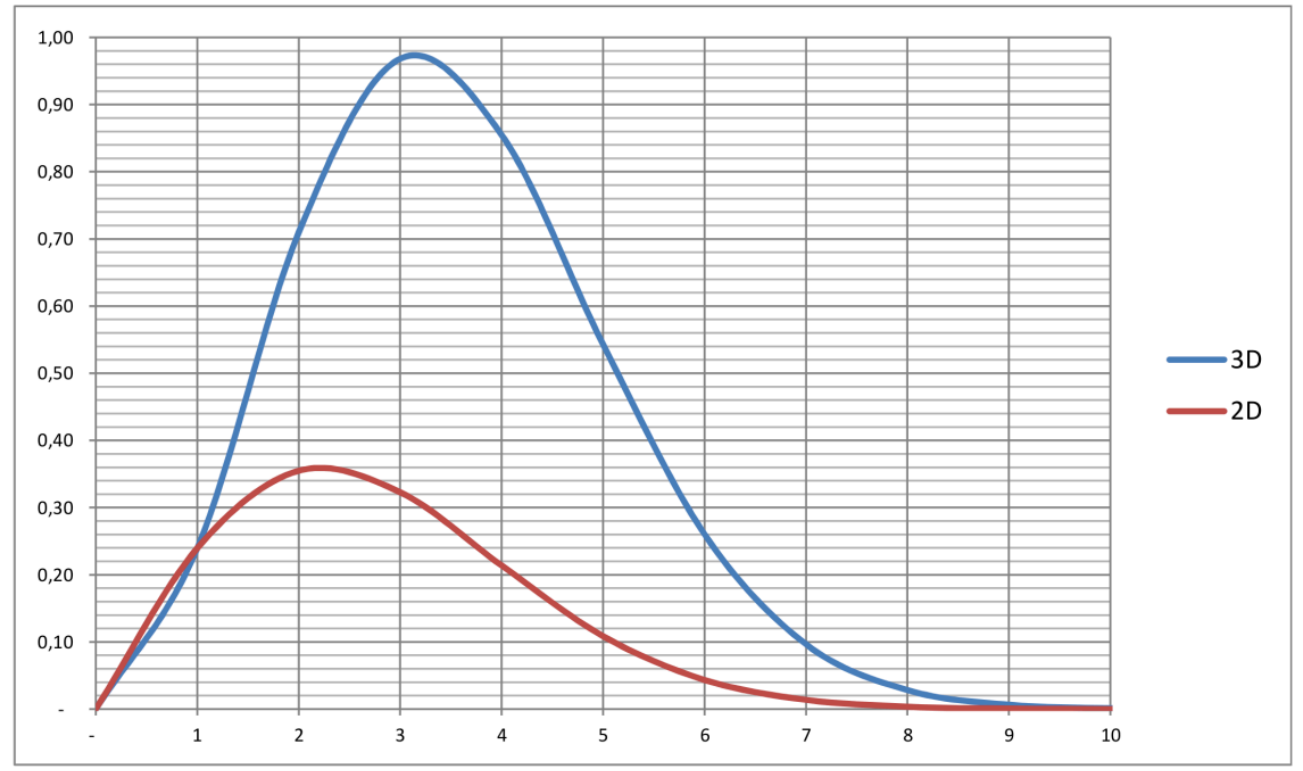

Fig. 3 - Comparação entre duas distribuições de Maxwell-Boltzmann com os mesmos parâmetros em duas e três dimensões. 


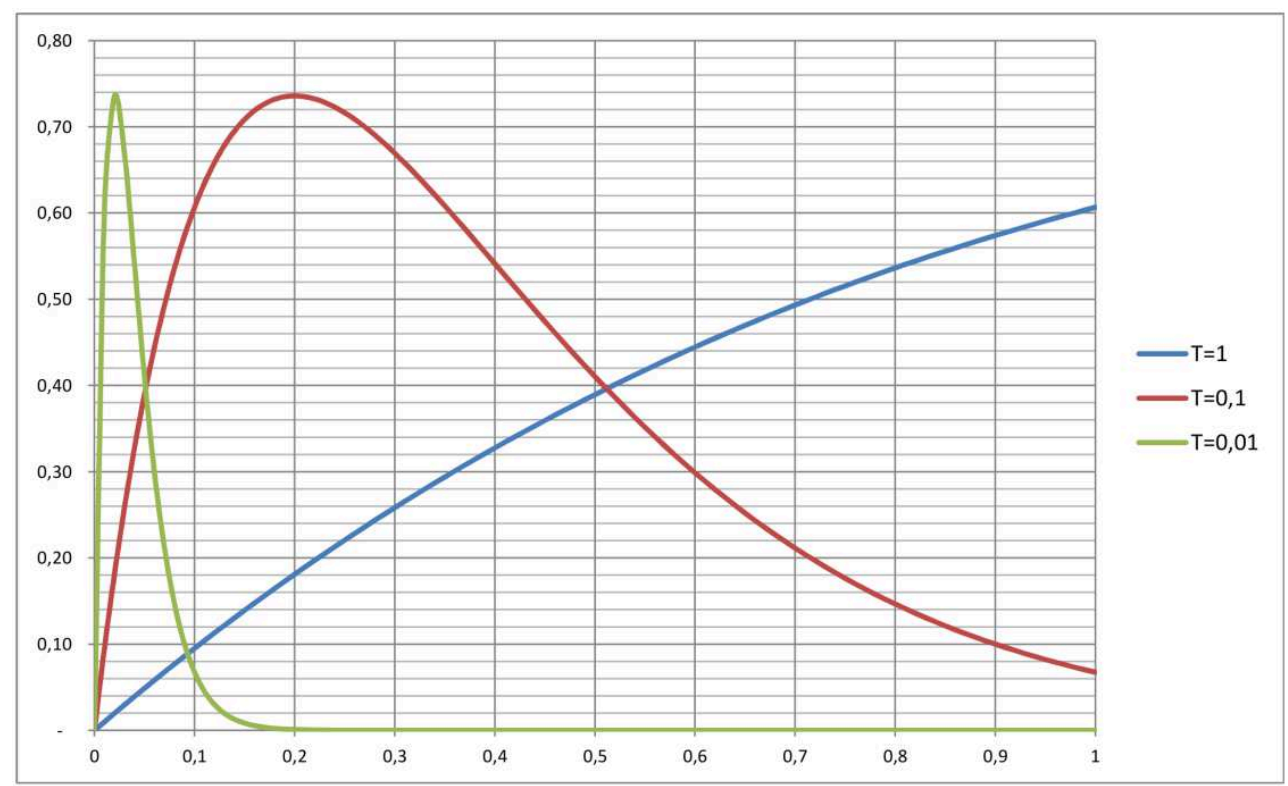

Fig. 4 - Gráfico da distribuição bidimensional dos módulos de velocidade de Maxwell-Boltzmann, para três diferentes valores de temperatura (Considerando $k=1$ ).

\section{Modelagem e Construção do Simulador}

Conforme as definições dadas para as equações de estado em 2D, o simulador terá um retângulo no qual as partículas serão consideradas como pontos que podem se movimentar livremente nas direções $x$ e $y$. Além da possibilidade do choque contra os lados do retângulo, as partículas podem colidir umas contra as outras. Estas colisões serão consideradas como perfeitamente elásticas, sendo válida a lei de conservação do momentum, pois as partículas também terão a propriedade de massa. A figura 5 mostra um exemplo de uma caixa mostrando as partículas em movimento dentro da mesma.

Quadro 1 - Resumo das equações de um gás ideal, considerando 2D e 3D. A diferença reside basicamente na consideração de área ao invés de volume e os fatores numéricos. (Observação: A pressão $P$ em 2D é análoga à mesma grandeza em 3D).

\begin{tabular}{|c|c|c|}
\hline Grandeza & 2D & 3D \\
\hline Energia cinética média & $\langle\epsilon\rangle=k T$ & $\langle\epsilon\rangle=\frac{3}{2} k T$ \\
\hline Pressão & $P=\frac{1}{2} \rho\left\langle v^{2}\right\rangle$ & $P=\frac{1}{3} \rho\left\langle v^{2}\right\rangle$ \\
\hline Equação de Clapeyron & $P A=n R T$ & $P V=n R T$ \\
\hline $\begin{array}{c}\text { Energia interna do gás } \\
\text { Velocidade quadrática } \\
\text { média }\end{array}$ & $U=P A$ & $U=\frac{3}{2} P V$ \\
\hline $\begin{array}{c}\text { Distribuição das velocida- } \\
\text { des }\end{array}$ & $p(v)=\frac{m}{k T} v \exp \left(-\frac{1}{2} \frac{m v^{2}}{k T}\right)$ & $p(v)=\sqrt{\frac{2}{\pi}\left(\frac{m}{k T}\right)^{\frac{3}{2}} v^{2} \exp \left(-\frac{1}{2} \frac{m v^{2}}{k T}\right)}$ \\
\hline
\end{tabular}


Para o contexto do simulador, as unidades de medida das grandezas envolvidas são modificadas conforme o modelo dado para a simulação. A medida de tempo, relacionada com cada passo da simulação, será o próprio passo. As medidas envolvendo distância serão dadas em pixels e a massa, temperatura e energia terão unidades padrão. Por questões de simplicidade, considera-se que a constante de Boltzmann $k$, a constante universal dos gases $R$ e o número de Avogadro tenham valor 1. Neste caso, a grandeza macroscópica da temperatura e a microscópica da energia cinética média das partículas serão iguais.

$$
\langle\epsilon\rangle=T
$$

A equação bidimensional de Clapeyron será dada por

$$
\frac{P A}{T}=n
$$

onde $n$ é agora o próprio número de partículas que compõem a simulação.

O simulador é construído na linguagem Javascript/HTML5, permitindo o acesso via browser $^{2}$, além da óbvia praticidade para a programação, pois não é necessária nenhuma ferramenta de desenvolvimento adicional exceto um editor de textos comum. Trabalhos sobre simulações físicas que utilizam bibliotecas específicas em Javascript podem ser encontrados na literatura (CLEMENTE, 2014; SILVA, 2016). Dentro da metodologia de programação orientada a objeto (ZAKAS, 2014) e com o uso da API Canvas para HTML5 (LUBBERS; ALBERS; SALIM, 2013, p. 25-63), é definida uma classe para as partículas, contendo propriedades tais como as coordenadas da posição $(x, y)$ dentro da área, a velocidade de cada componente $(d x, d y)$ e a massa $m$ da partícula:

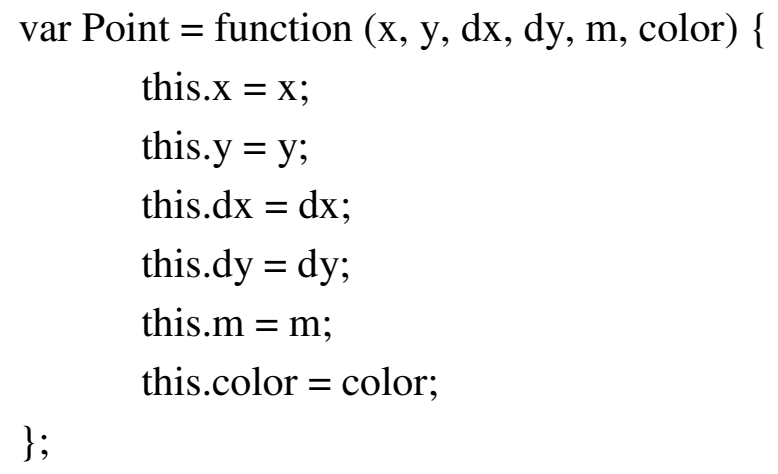

Para a classe da partícula é definido o método de movimento em um passo, fazendose o teste de colisão com as paredes da caixa:

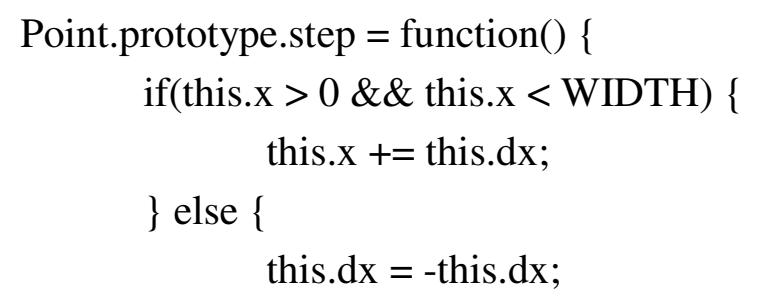

\footnotetext{
${ }^{2} \mathrm{O}$ simulador pode ser acessado no endereço: <http://93.188.163.112/projects/animation.html>.
} 


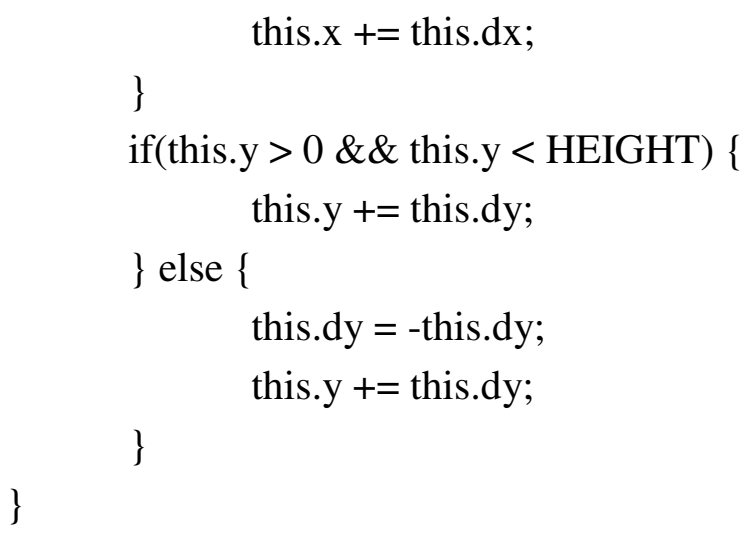

No caso de colisão com outra partícula, considera-se a transferência de momentum, conforme o teste da distância entre elas abaixo de um determinado limiar:

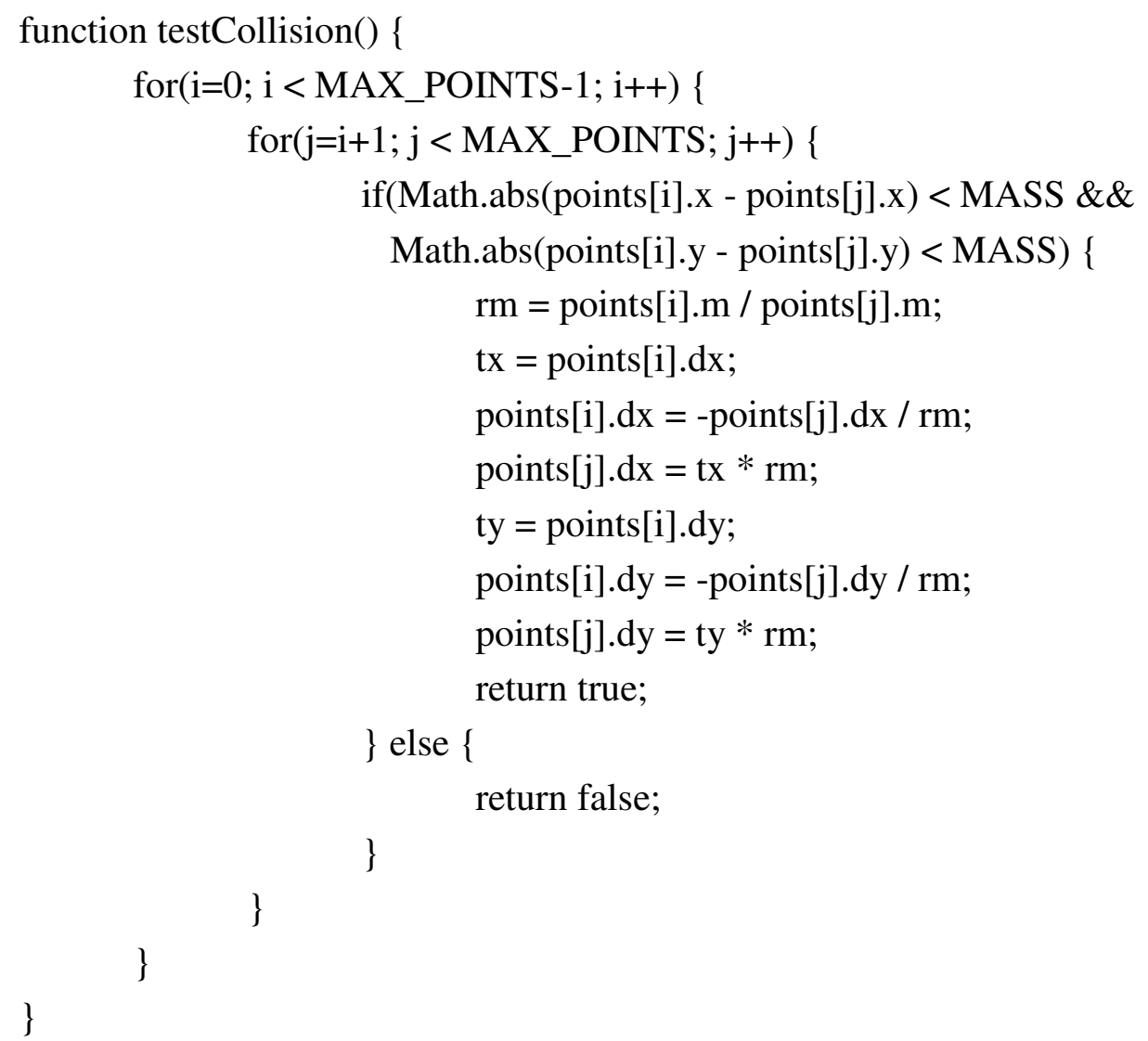

Antes da execução, os parâmetros iniciais são fornecidos: as dimensões da área, o número de partículas e a massa de cada partícula, bem como a velocidade inicial das partículas, sendo esta um valor médio para a geração de velocidades aleatórias das partículas. As grandezas referentes à equação de estado são calculadas: a velocidade quadrática média das partículas, pressão, a densidade, e a temperatura. As velocidades de cada partícula são plotadas em um histograma, indicando a distribuição de velocidades.

A partir da execução do simulador, o usuário pode acompanhar a cada passo a posição das partículas (Fig. 5), a definição e o cálculo das grandezas (Fig. 6) e o histograma para cada execução (Fig. 7), conforme os parâmetros alimentados à simulação. 


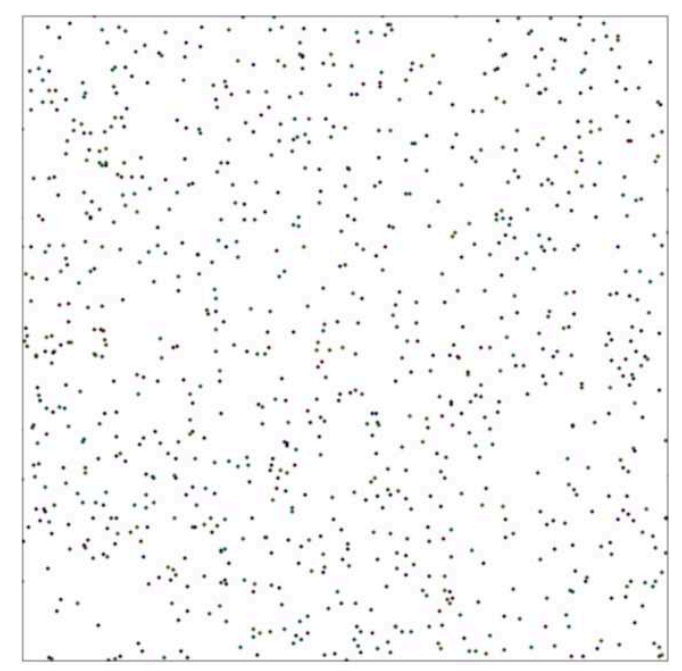

Fig. 5 - Caixa em duas dimensões com 1000 partículas em movimento livre e considerando colisões perfeitamente elásticas.

\begin{tabular}{|c|c|}
\hline Quantity & Value \\
\hline Num.of Points & 1000 \\
\hline Side & 500 \\
\hline Initial random base velocity (pixels/step) & 50 \\
\hline Mass (standard-mass) & 1 \\
\hline Mean square velocity (pixels ${ }^{2} /$ step $^{2}$ ) & 1662.24 \\
\hline Mean velocity (pixels/step) & 40.77 \\
\hline Density (standard-mass/pixels ${ }^{2}$ ) & 0.004 \\
\hline Pressure (standard-mass.pixels/step ${ }^{2}$.pixels ${ }^{2}$ ) & 3.324 \\
\hline Area $\left(\right.$ pixels $\left.{ }^{2}\right)$ & 250000 \\
\hline Temperature (standard-temperature) & 831.12 \\
\hline $\mathrm{P} * \mathrm{~A} / \mathrm{T}(=\mathrm{n})$ & 1000 \\
\hline
\end{tabular}

Fig. 6 - Tabela com os parâmetros e as grandezas calculadas para cada execução no simulador. As grandezas estão medidas em unidades conforme definidas no quadro 1. Após o cálculo da equação de Clapeyron, obtém-se novamente o número de partículas fornecido no início da simulação. 


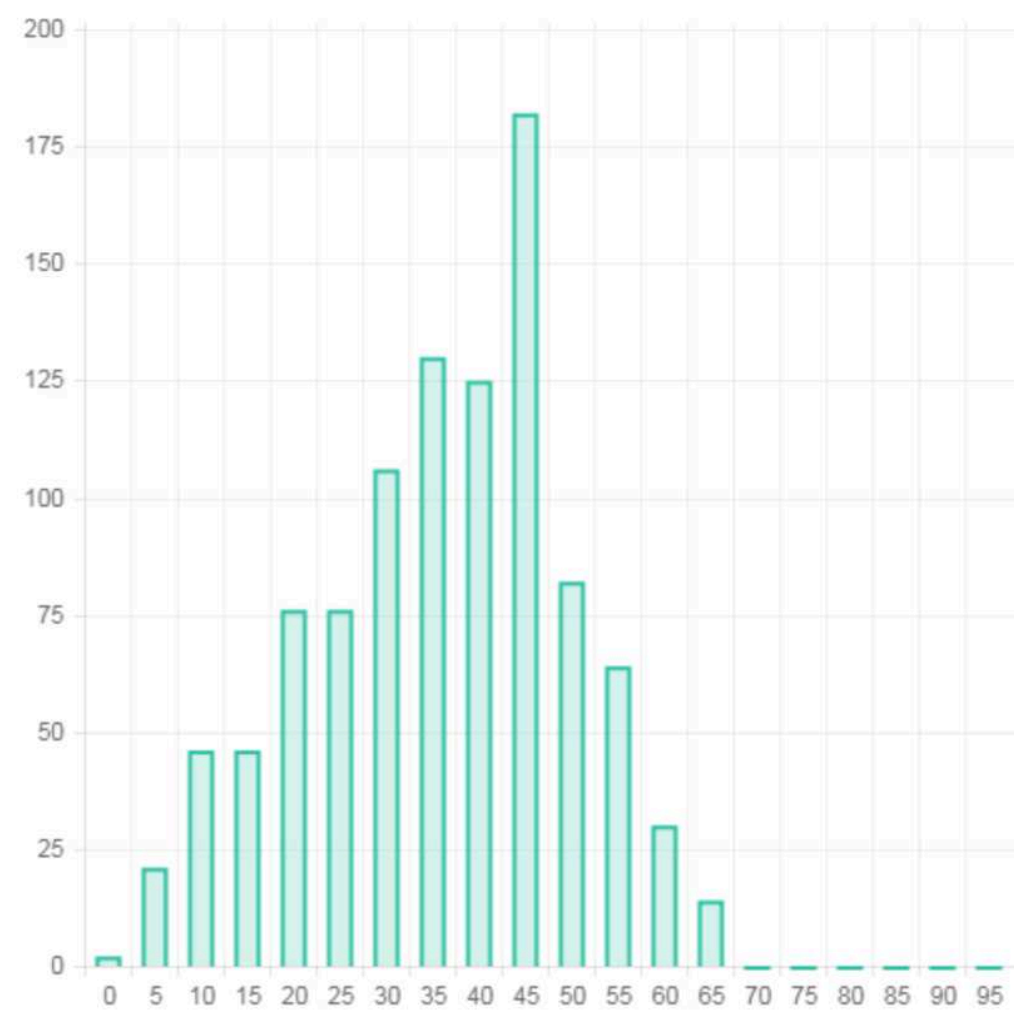

Fig. 7 - Histograma das velocidades médias de uma simulação específica, mostrando aproximação com a curva da distribuição de Maxwell-Boltzmann, conforme visualizada na Fig. 4.

Para a mensuração das grandezas no contexto da simulação, foram estabelecidas as unidades de medida conforme mostra o quadro 2.

Quadro 2 - Definição das unidades de medida para as grandezas consideradas no simulador.

\begin{tabular}{|c|c|c|}
\hline Grandeza/Constante & Símbolo & Unidade de medida \\
\hline Massa & $m$ & massa-padrão \\
\hline Tempo & $t$ & passo \\
\hline Área & $A$ & pixels $^{2}$ \\
\hline Velocidade & $v$ & pixels/passo \\
\hline Aceleração & $a$ & pixels/passo ${ }^{2}$ \\
\hline Temperatura & $T$ & temperatura-padrão \\
\hline Força & $F$ & massa-padrão.pixels/passo ${ }^{2}$ \\
\hline Pressão & $P$ & massa-padrão.pixels/passo ${ }^{2}$.pixels ${ }^{2}$ \\
\hline Densidade & $\rho$ & massa-padrão/pixels ${ }^{2}$ \\
\hline Energia & $\epsilon$ & energia-padrão \\
\hline Constante $\mathrm{k}$ & $k$ & energia-padrão.temperatura-padrão ${ }^{-1}$ \\
\hline Constante R & $R$ & energia-padrão.temperatura-padrão ${ }^{-1}$ \\
\hline
\end{tabular}


Com relação aos simuladores semelhantes citados, Aiello-Nicosia e SperandeoMineo (1985) utilizaram uma metodologia semelhante, adotando colisões elásticas para partículas idênticas com mesmo diâmetro, demonstrando a distribuição de velocidades próxima à previsão dada pela distribuição de Maxwell-Boltzmann em 2D. Entretanto, o número de partículas utilizado nas simulações, conforme relatado, foi de somente 16. Bonomo e Riggi (1984) utilizaram simulações de 20.000 partículas idênticas como discos rígidos, sendo as colisões elásticas e comparando a distribuição de velocidades ao final da simulação. Outro resultado interessante noticiado neste trabalho foi a aproximação gradativa da distribuição teórica de Maxwell-Boltzmann com o aumento do número de colisões entre as partículas. O simulador aqui descrito permitiu utilizar 20.000 partículas, demonstrando um desempenho razoável (4,7 segundos/passo) em um computador com processador Intel ${ }^{\circledR}$ Core $^{\mathrm{TM}}$ i7 e 8 GB de RAM, mostrando em tempo real na página da simulação o histograma de velocidades, tal como o apresentado na Fig. 7, a cada passo.

\section{Analisando as colisões com as bordas}

Para a geração das posições e velocidades iniciais das partículas na simulação, utiliza-se a função de geração de números aleatórios. Como o gerador implementado no Javascript retorna valores em ponto flutuante na faixa $[0,1]$, a obtenção das coordenadas $(x, y)$ de uma dada partícula é feita multiplicando-se o valor obtido para uma dada partícula pelas dimensões respectivas a cada coordenada da caixa. Desta forma, tem-se um espalhamento uniforme das partículas nas condições iniciais da simulação.

Com relação à velocidade, a partir de uma velocidade inicial $v_{i}$ informada pelo usuário, as componentes de velocidades são calculadas de forma que a partícula esteja em direções aleatórias no início da simulação. Se $r$ pertence a $[0,1]$, o valor aleatório gerado no momento da atribuição das velocidades, a velocidade obtida para cada componente, com $r$ gerado especificamente para cada uma, é dada por

$$
\begin{aligned}
& v_{x}=v_{i}(1-2 r) \\
& v_{y}=v_{i}(1-2 r)
\end{aligned}
$$

Isto garante que $v_{x}$ ou $v_{y}$ estejam dentro da faixa de valores $\left[-v_{i}, v_{i}\right]$, e que as velocidades iniciais sejam suficientemente aleatórias em diversas direções. Outra maneira é proporcionar que a geração de números aleatórios siga a distribuição de Maxwell-Boltzmann. Entretanto, mesmo utilizando este tipo de geração de números aleatórios, as análises das distribuições de velocidades tendem sempre para uma distribuição aproximada de MaxwellBoltzmann, como já apontado anteriormente por Bonomo e Riggi (1984, p. 55).

A partir de uma análise experimental, um efeito interessante pode ser identificado quando se verifica a quantidade de colisões das partículas com as paredes da caixa. A simulação se inicia com um determinado valor e as colisões com as bordas vão aumentando à medi- 
da que aumentam os passos da simulação. Apesar das condições iniciais serem geradas de forma aleatória, tem-se a impressão de que o gás bidimensional precisa alcançar uma espécie de configuração mais estável quanto às colisões com as bordas, uma condição que não é proporcionada somente com a aleatoriedade no início da simulação.

Como mostrado na Fig. 5, quando a partícula alcança alguma borda da caixa, ela é refletida, tendo a mudança de sinal na componente, seja em $x$ ou $y$, mas mantendo-se o mesmo valor. O gráfico da Fig. 8 mostra o comportamento assintótico do número de colisões com as bordas em uma dada simulação após aproximadamente 500.000 passos. Apesar da tendência de crescimento, é esperado que o número de colisões se estabilize em torno de um determinado valor para manter a uniformidade na distribuição das partículas na caixa. Isto pode ser constatado em simulações com menor número de partículas, com o gráfico bastante semelhante ao comportamento assintótico da função erro.

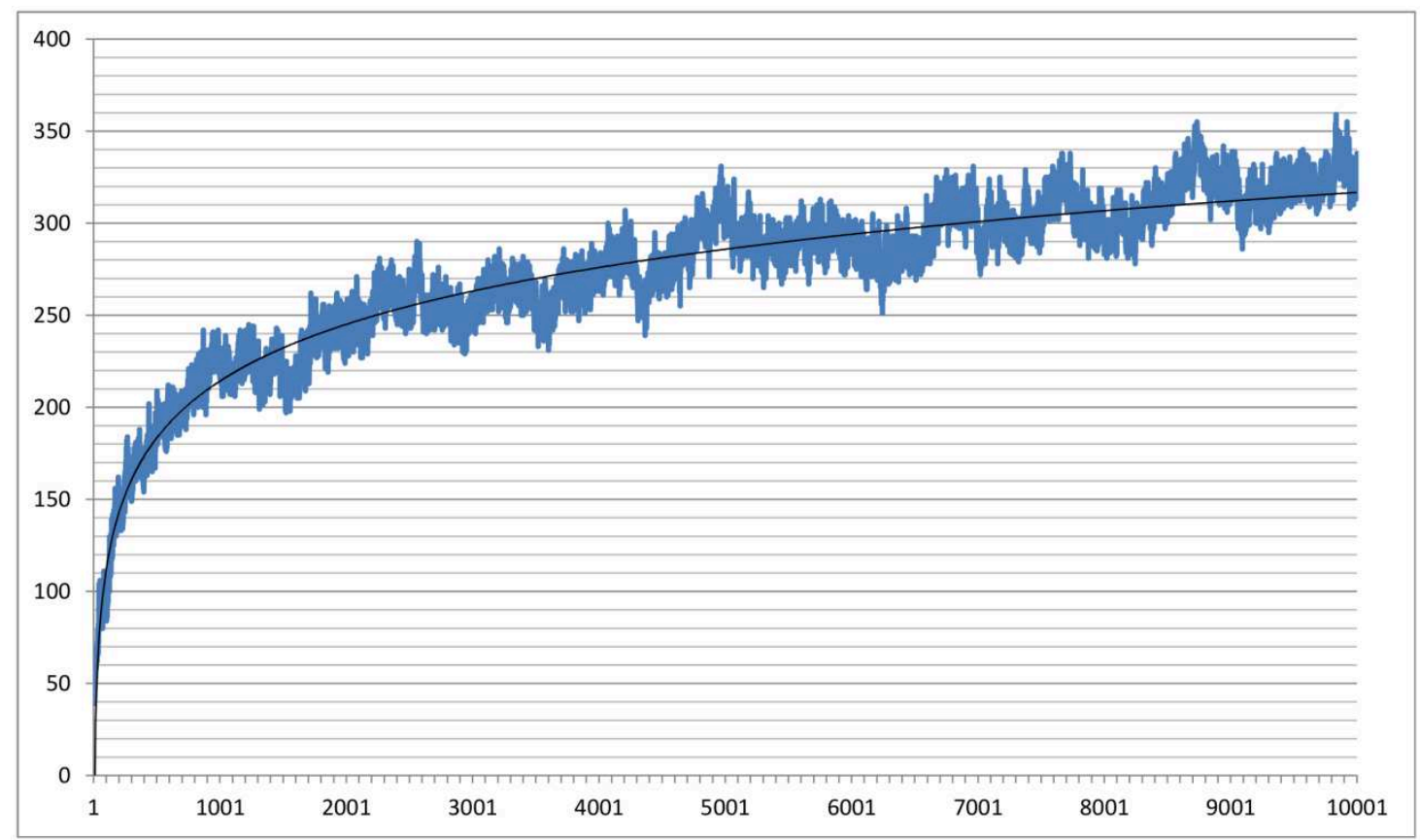

Fig. 8 - Número de colisões das partículas com as bordas da caixa, mostrando um comportamento assintótico crescente. A escala de passos é de 1:50.

Experimentalmente, observa-se que o número de colisões com as bordas é proporcional à quantidade de partículas e à velocidade das mesmas. Na Fig. 9 pode-se identificar, para 4 diferentes isotermas, o crescimento da contagem de colisões (apenas com as bordas) com o aumento do número de partículas. 
Quadro 3 - Análise de regressão da relação entre a quantidade de partículas e o número de colisões com as paredes, com base em uma função do tipo $\mathrm{y}=\mathrm{ax}$.

\begin{tabular}{|l|c|c|c|}
\hline \multicolumn{1}{|c|}{$T$} & $a$ & $b$ & $R^{2}$ \\
\hline 50 & 0,0043 & 1,4556 & 0,9961 \\
\hline 200 & 0,0096 & 1,4167 & 0,9999 \\
\hline 800 & 0,0181 & 1,3783 & 0,9996 \\
\hline 1800 & 0,0255 & 1,3539 & 0,9997 \\
\hline
\end{tabular}

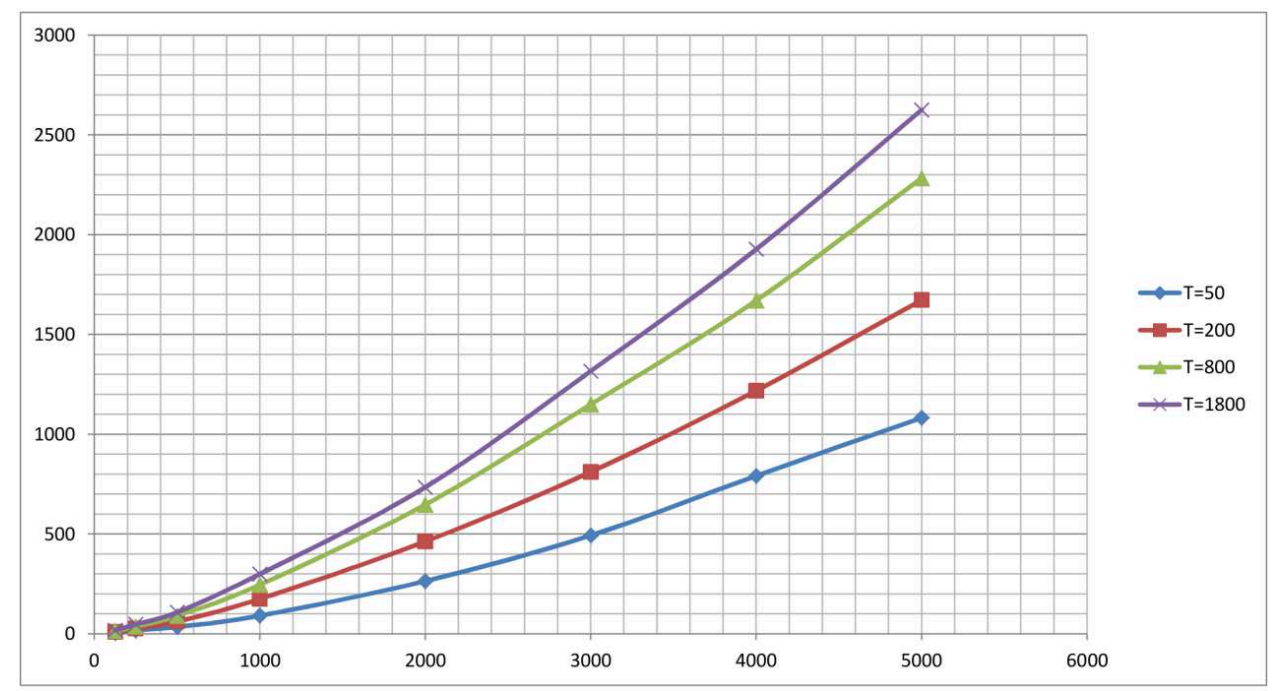

Fig. 9 - Gráfico do número de partículas (eixo horizontal) pela quantidade de colisões das partículas com as bordas da caixa (eixo vertical).

Uma análise preliminar de regressão mostra alta correlação das isotermas conforme o quadro 3, considerando-se uma função do tipo $y=a x^{b}$.

Conjectura-se aqui que este efeito de nível microscópico possa interferir nos resultados de simulações mais elaboradas, que contemplem as transformações relativas às grandezas macroscópicas. Por exemplo, considerando uma simulação da lei de decaimento exponencial da temperatura de Newton, no qual as colisões das partículas com as bordas seriam inelásticas, iniciar o processo de decaimento no início da simulação produzirá um comportamento diferente caso se inicie a partir de um número maior de iterações.

Por fim, esta análise das colisões, além de fornecer algumas características quanto ao comportamento das partículas confinadas em $2 \mathrm{D}$, pode também ser utilizada como um importante subsídio para fins de ensino e aprendizagem. Os dados para a análise foram coletados a partir de diversas execuções do simulador com diferentes parâmetros. Dessa forma, como objeto de aprendizagem, o simulador pode ser utilizado como ferramenta para pesquisa exploratória sobre o comportamento do fenômeno. 


\section{Considerações finais}

Os resultados obtidos com o simulador estiveram em consonância com aqueles descritos nos simuladores citados nas referências, permitindo chegar a conclusões semelhantes. Algumas observações podem ser feitas a partir da execução das simulações. Uma é o próprio cálculo das grandezas que leva à obtenção do número de partículas, a partir da equação de Clapeyron, exatamente como fornecido no parâmetro. A partir da execução das simulações, as partículas apresentam colisões elásticas, sendo que as grandezas macroscópicas não se modificam em função da suposição do gás ideal, tendo todas as partículas a mesma massa. Entretanto, o aspecto mais relevante é a própria constatação de que variáveis macroscópicas termodinâmicas são obtidas a partir de variáveis microscópicas sujeitas às leis newtonianas, mostrando a relação inerente aos níveis físicos micro e macroscópico.

Portanto, este artigo teve a intenção de demonstrar, por meio do simulador, a visualização da transição entre o fenômeno microscópico, a partir das colisões elásticas seguindo a mecânica newtoniana, e a emergência das grandezas macroscópicas relacionadas com o fenômeno termodinâmico. Ainda que seja com a suposição de um gás ideal em duas dimensões, o experimento permite a compreensão do argumento teórico por trás da ideia revolucionária do século XIX sobre o comportamento de um gás estar relacionado intimamente ao movimento dinâmico das moléculas que o compõem.

Caruso e Oguri (2006, p. 67-68) destacam que a teoria cinética dos gases, além de marcar o apogeu da mecânica de Newton, foi bem sucedida na explicação de vários fenômenos e terminaram por conduzir à concepção majoritária de que a matéria é constituída fundamentalmente de átomos e moléculas. $\mathrm{O}$ estudo da distribuição de Maxwell-Boltzmann serve também como ponto de partida para as distribuições de Fermi-Dirac e de Bose-Einstein (EISBERG; RESNICK, 1979, p. 479).

Além dos desdobramentos e possibilidades de estudos descritos na seção da análise das colisões com as bordas, versões futuras contemplarão a evolução do simulador para explicação de fenômenos considerando misturas de gases com diferentes massas moleculares para demonstração da evolução entrópica, paredes divisórias para simulação de processos de separação de gases e a explicação de um processo hipotético de um "gênio" de Maxwell fazendo a separação de gases e seu reflexo no cálculo da entropia do sistema.

\section{Referências}

AIELlO-NICOSIA, M. L.; SPERANDEO-MINEO, R. M. Computer simulation of a twodimensional ideal gas: a simple molecular dynamics method for teaching purposes. European Journal of Physics, v. 6, n. 3, p. 148, 1985.

BONOMO, R. P.; RIGGI, F. The evolution of the speed distribution for a two-dimensional ideal gas: A computer simulation. American Journal of Physics, v. 52, n. 1, p. 54-55, 1984. 
BREY, J. J.; MAYNAR, P.; GARC, M. I. Kinetic equation and nonequilibrium entropy for a quasi-two-dimensional gas. Physics Review E 94, v. 40103, p. 1-4, 2016.

CARUSO, F.; OGURI, V. Fìsica Moderna: Origens clássicas e fundamentos quânticos. Rio de Janeiro: Elsevier, 2006.

CLEMENTE, F. J. G. EjsS: A JavaScript library which makes computational-physics education simpler. XXVI IUPAP Conference on Computational Physics. Anais... BostonMA: 2014

EISBERG, R.; RESNICK, R. Física Quântica: Átomos, Moléculas, Sólidos, Núcleos e Partículas. Rio de Janeiro: Editora Campus, 1979.

LUBBERS, P.; ALBERS, B.; SAlIM, F. Programação Profissional em HTML5. Rio de Janeiro: Books, Alta, 2013.

MEDEIROS, A.; MEDEIROS, C. F. DE. Possibilidades e Limitações das Simulações Computacionais no Ensino de Física. Revista Brasileira de Ensino de Física, v. 24, n. 2, p. 77-86, 2002.

NOVAK, J.; BORTZ, A. B. The Evolution of the Two-Dimensional Maxwell-Boltzmann Distribution. American Journal of Physics, v. 38, n. 12, p. 1402-1406, 1970.

NOVOSELOV, K. S. et al. A roadmap for graphene. Nature, v. 490, n. 7419, p. 192-200, 2012.

RESNICK, R.; HALLIDAY, D. Física II. 3. ed. Rio de Janeiro: LTC, 1982.

SCHERER, C. Métodos Computacionais em Física. São Paulo: Livraria da Física, 2005.

SILVA, N. C. DA. Física Moderna e Técnicas Computacionais: Como "ver" o Átomo de Hidrogênio. Caderno Brasileiro de Ensino de Física, v. 33, n. 2, p. 667-696, 2016.

STRANICK, S. J.; KAMNA, M. M.; WEISS, P. S. Atomic Scale Dynamics of a TwoDimensional Gas-Solid Interface. Science, v. 266, n. 5182, p. 99-102, 1994.

TIPLER, P. A.; MOSCA, G. Física para Engenheiros e Cientistas. 6. ed. Rio de Janeiro: LTC, 2014.

ZAKAS, N. C. Princípios de Orientação a Objetos em Javascript. São Paulo: Novatec, 2014.

(c) BY-NC-ND Direito autoral e licença de uso: Este artigo está licenciado sob uma Licença Creative Commons. 